\title{
Troglitazone reverses the multiple drug resistance phenotype in cancer cells
}

\author{
Gerald F Davies' \\ Bernhard HJ Juurlink ${ }^{2}$ \\ Troy AA Harkness' \\ 'Department of Anatomy \\ and Cell Biology, College \\ of Medicine, University \\ of Saskatchewan, Saskatoon, \\ Canada; ${ }^{2}$ College of Medicine, \\ Alfaisal University, Riyadh, \\ Kingdom of Saudi Arabia
}

Correspondence: Troy AA Harkness Department of Anatomy and Cell Biology, College of Medicine, University of Saskatchewan, 107 Wiggins Road, Saskatoon, SK, Canada, S7N 5E5

$\mathrm{Tel}+\mathrm{I} 3069661995$

Fax +I 3069662542

Email troy.harkness@usask.ca

\begin{abstract}
A major problem in treating cancer is the development of drug resistance. We previously demonstrated doxorubicin (DOX) resistance in K562 human leukemia cells that was associated with upregulation of glyoxalase 1 (GLO-1) and histone $\mathrm{H} 3$ expression. The thiazolidinedione troglitazone (TRG) downregulated GLO-1 expression and further upregulated histone $\mathrm{H} 3$ expression and post-translational modifications in these cells, leading to a regained sensitivity to DOX. Given the pleiotropic effects of epigenetic changes in cancer development, we hypothesized that TRG may downregulate the multiple drug resistance (MDR) phenotype in a variety of cancer cells. To test this, MCF7 human breast cancer cells and K562 cells were cultured in the presence of low-dose DOX to establish DOX-resistant cell lines (K562/DOX and MCF7/DOX). The MDR phenotype was confirmed by Western blot analysis of the $170 \mathrm{kDa}$ P-glycoprotein (Pgp) drug efflux pump multiple drug resistance protein 1 (MDR-1), and the breast cancer resistance protein (BCRP). TRG markedly decreased expression of both MDR-1 and BCRP in these cells, resulting in sensitivity to DOX. Silencing of MDR-1 expression also sensitized MCF7/DOX cells to DOX. Use of the specific and irreversible peroxisome proliferator-activated receptor gamma (PPAR $\gamma$ ) inhibitor GW9662 in the nanomolar range not only demonstrated that the action of TRG on MCF/DOX was PPAR $\gamma$-independent, but indicated that PPAR $\gamma$ may play a role in the MDR phenotype, which is antagonized by TRG. We conclude that TRG is potentially a useful adjunct therapy in chemoresistant cancers.
\end{abstract}

Keywords: chemotherapy, doxorubicin, breast cancer resistance protein-1, multiple drug resistance, multiple drug resistance protein 1

\section{Introduction}

Chemotherapy is an important tool in both the initial treatment of many cancers, ${ }^{1}$ as well as in treating metastatic cancer. ${ }^{2}$ However, a large proportion of patients either do not respond to chemotherapy or lose their initial responsiveness. ${ }^{2}$ Breast cancer is a major worldwide health issue for women, and has often already metastasized by the time of initial diagnosis. ${ }^{3}$ Every year, 1,000,000 new cases are diagnosed, with 40,000 deaths in the United States alone every year. The response rates of metastatic breast cancer to initial treatment ranges from $30 \%-70 \%$, and is frequently temporary, as time to disease progression following these treatments is only 6-10 months. The response rate for second round chemotherapy for those whose disease progresses falls to $20 \%-30 \%$ with a median duration of response less than six months. This failure to respond to chemotherapy is primarily due to drug resistance mechanisms. The most common mechanism of drug resistance is expression of the drug efflux pumps belonging to the adenosine triphosphate (ATP)-binding cassette (ABC) transporter family. ${ }^{2}$ One of the most commonly expressed of these transporters is the multiple drug resistance protein 1 (MDR-1; or ABCB1) gene that encodes for P-glycoprotein (Pgp). For example, approximately 50\% of all breast cancers have been reported to express the MDR-1 gene ${ }^{4}$ and resistance to chemotherapy correlates with MDR-1 expression. ${ }^{5,6}$ There is now a concerted effort to develop drugs that can overcome multiple drug resistance (MDR). ${ }^{7,8}$ 
We had previously shown that the peroxisome-proliferator activated receptor gamma (PPAR $\gamma$ ) agonist troglitazone (TRG), but not other thiazolidinediones, downregulated glyoxalase I gene expression in a variety of cell types. ${ }^{9}$ Since overexpression of glyoxalase $I$ is present in a number of cancers, increased expression of glyoxalase I is thought to contribute to drug resistance..$^{10,11} \mathrm{We}$ had hypothesized that TRG would be capable of downregulating glyoxalase I gene expression in tumor cells and sensitize these cells to therapeutic intervention. Indeed, our subsequent experiments did show that TRG downregulated glyoxalase I and did overcome doxorubicin (DOX) resistance in DOX-resistant K562 human leukemia cells. ${ }^{12}$ These results led us to hypothesize that TRG may also downregulate MDR-1 gene expression in cancer cells. Here, we present data that TRG does indeed downregulate increased expression of MDR-1 in DOX-resistant K562 leukemia cells and MCF7 human breast cancer. Our results suggest TRG may be a useful adjunct therapy in treating a variety of cancer cells.

\section{Materials and methods \\ Cell culture}

MCF7 human breast cancer cells, as well as K562 human leukemia cells (obtained from American Type Culture Collection), were cultured in $75 \mathrm{~cm}^{2}$ tissue culture flasks (Falcon) in Dulbecco's Modified Eagle's Medium (DMEM-F12) and RPMI 1640 media, respectively, containing 10\% fetal bovine serum (Invitrogen, Carlsbad, CA, USA). Cells were cultured in a humidified atmosphere $\left(5 \% \mathrm{CO}_{2}\right)$ at $37^{\circ} \mathrm{C}$ and at appropriate times were incubated for $48 \mathrm{~h}$ in the presence of the compounds at the indicated concentrations.

\section{DOX-resistant cell selection}

Parental MCF7 and K562 cells were cultured in the presence of $1 \mu \mathrm{M}$ DOX over a period of $72 \mathrm{~h}$. At the end of this period the cells were washed with sterile phosphate-buffered saline (PBS) to remove the DOX and fresh DOX-free growth medium was added. Following a month-long recovery period (with fresh medium changes every 3 days) $1 \mu \mathrm{M}$ DOX was again added to the cells for $72 \mathrm{~h}$. Following a PBS wash $10 \mathrm{nM}$ DOX was added to the medium to maintain selection pressure. These selected mutant lines were designated MCF7/DOX and K562/DOX, respectively. Selected cells were washed free of any DOX prior to experimentation. Selected MCF7/DOX maintained their MDR phenotype after long-term storage in liquid nitrogen without requiring re-selection.
Cultures were treated with one of the following: $1 \mu \mathrm{M}$ DOX, 50 or $100 \mu \mathrm{M}$ TRG, $1 \mu \mathrm{M}$ DOX plus $50 \mu \mathrm{M}$ TRG or simply the dimethylsulfoxide vehicle (DMSO: final concentration of $0.1 \%$ ) in which DOX and TRG was prepared.

\section{Western blot analysis}

Breast cancer cells were removed from the culture dishes using a rubber policeman and ice-cold lysis buffer. K562 cells, which were either loosely adherent or floating, were removed in their medium, centrifuged at $300 \times \mathrm{g}$ and resuspended in ice-cold lysis buffer. The composition of the lysis buffer was as follows: $20 \mathrm{mM}$ HEPES (pH 7.5), $50 \mathrm{mM} \mathrm{KCl}, 10 \%$ glycerol, 0.5 mM EDTA, 0.1 mM EGTA, $1 \mathrm{mM}$ DTT plus $1 \times$ mammalian cell anti-protease cocktail (Sigma Aldrich Canada, Oakville, ON, Canada). The cells were lysed using multiple freeze-thaw cycles followed by pulse sonication and centrifugation at $300 \times \mathrm{g}$ to remove nonlysed cell debris. For Western blot analysis, equivalent amounts of protein (assessed by Bradford protein assay ${ }^{13}$ using BioRad Protein Reagent; BioRad Laboratories, Hercules, CA, USA) were resolved by sodium dodecyl sulfate-polyacrylamide gel electrophoresis (SDS-PAGE). Following electrophoresis the proteins were transblotted onto nitrocellulose membranes (Pall-VWR). The membranes were blocked with 5\% molecular grade fat free skim milk powder (BioRad Laboratories) in phosphatebuffered saline (PBS) containing 0.1\% Tween-20 (PBST). Primary and secondary antibody incubations and subsequent washes were carried out in the same buffer. Secondary horseradish peroxidase (HRP) antibodies were purchased from BioRad Laboratories. Blots were blocked overnight at $4{ }^{\circ} \mathrm{C}$ on a gyratory plate and probed with primary antibodies at a 1:1000 dilution overnight. Secondary HRP antibody was applied at room temperature on a gyratory plate at a concentration of 1:10,000 for $30 \mathrm{~min}$. Following washes, an enhanced chemiluminescence detection system (DuPont-NEN ${ }^{\circledR}$; DuPont, Boston, MA, USA) was used to detect the antigen/antibody complexes. Blots were then stripped and reprobed for either actin, $\alpha$-tubulin or glyceraldehyde-3-phosphate dehydrogenase (GAPDH) as protein load controls. Alternatively, Coomassie-stained gels were shown as load controls. Primary antibodies to alpha-tubulin and actin were obtained from Sigma Aldrich and Pgp antibody was purchased from Santa Cruz Biotechnology (Santa Cruz, CA, USA). Antibody to breast cancer resistance protein 1 (BCRP1) was obtained from Abcam (Cambridge, MA, USA). 


\section{MTT cell viability assay}

Determination of cell viability was accomplished by the MTT assay. The MTT (3-(4,5-dimethylthiazol-2-yl)2,5-diphenyltetrazolium bromide) assay is a colorimetric method that is widely used to determine viability of eukaryotic cells following drug treatment. ${ }^{14}$ This assay is based upon the reduction of MTT (a yellow compound) to a purple formazan in the mitochondria of living, viable cells. Cancer cell suspensions were prepared at a concentration of approximately $10^{6}$ cells per $\mathrm{ml}$ as determined by standard hemocytometry and cultured in six-well multiwell plates. These cultures were treated simultaneously with cells cultured in $75 \mathrm{~cm}^{2}$ tissue culture flasks dedicated to protein extraction and Western blot analysis. For MTT analysis cells were cultured in phenol red-free medium to avoid interfering with the colorimetric analysis of the purple formazen MTT product. Following $48 \mathrm{~h}$ of treatment cells were treated with MTT followed by washing, dodecyl sulfate (SDS) solubilization of the formazan product and spectrometric analysis at $570 \mathrm{~nm}$.

\section{RNA silencing}

MCF7/DOX cells $\left(10^{6}\right)$ were transfected with $1 \mu \mathrm{g}$ of duplex human MDR-1 small interfering RNA (siRNA) following the manufacturers instructions (Santa-Cruz Biotechnology). Briefly, cells were cultured in $100 \mathrm{~mm}$ tissue culture plates and washed with PBS, followed by serum-free and antibiotic-free DMEM prior to transfection. siRNA duplex solutions (fluorescein-conjugated, scrambled siRNA control and MDR-1 siRNAs) were prepared by adding $1 \mu \mathrm{g}$ (80 pmols) of MDR-1 siRNA to the supplied transfection reagent (sc-29528). The resulting reaction mixture was allowed to complex for 30 minutes at room temperature. Next, the mixture was gently overlayed onto the cells, and incubated for six hours at $37^{\circ} \mathrm{C}$ in a $\mathrm{CO}_{2}$ incubator. Following this incubation period, an equivalent volume of $2 \times$ growth media ( $20 \%$ fetal bovine serum [FBS] and $2 \times$ antibiotics) was added without removing the transfection mixture, and the cells were incubated overnight. The next morning the cells were washed once with phosphate-buffer solution (PBS) and resuspended in $1 \times$ growth media. Incubation of the siRNA transfected cells was continued and analysis was performed after $72 \mathrm{~h}$. Fluorescein conjugated scrambled control siRNA treated cells were quantified using fluorescence microscopy (Olympus, Lehigh Valley, PA) to determine transfection efficiency and whole cell lysates of both scrambled and MDR-1 siRNA-treated cells were prepared for MDR-1 Western analysis.

\section{Reagents}

TRG was purchased from Biomol Laboratories (Plymouth Meeting, PA, USA). DMEM-F12 media was purchased from Sigma Aldrich Canada. Fetal bovine serum and antibiotics were purchased from Invitrogen. Molecular biology grade skim milk powder was purchased from BioRad Laboratories Canada (Mississauga, ON) while DOX and all other reagents were purchased from Sigma Aldrich Canada.

\section{Statistical analysis}

Data from one cell type were examined with a one-way ANOVA and post-hoc Tukey-Kramer Multiple Comparisons test. Data between specific cell types of any given treatment were examined with the nonparametric Mann-Whitney test.

\section{Results}

Unselected MCF7 cells expressed a low basal level of the ATP-transporters MDR-1 and BCRP (Figure 1A). DOX selection resulted in elevated MDR-1 and BCRP signals in MCF7 cells. Next, we tested whether treatment of DOXselected cells with TRG would decrease expression of MDR-1 and BCRP. As with the GLO-1 downregulation we observed earlier, ${ }^{12} 50 \mu \mathrm{M}$ TRG downregulated MDR-1 in MCF7 cells (Figure 1A). For BCRP downregulation, $100 \mu \mathrm{M}$ TRG was required rather than $50 \mu \mathrm{M}$ TRG for MDR-1 downregulation. We also observed downregulation of MDR-1 and BCRP by $50 \mu \mathrm{M}$ TRG in F98 rat glioblastoma cells and H411E rat hepatoma cells (data not shown). Use of TRG at $50-100 \mu \mathrm{M}$ is well above the half maximal inhibitory concentration $\left(\mathrm{IC}_{50}\right)$ of TRG for PPAR $\gamma$ which is $0.42 \mu \mathrm{M}$ in rat adipocytes and $1.05 \mu \mathrm{M}$ in human adipocytes. ${ }^{15}$ Nonetheless, primary hepatocytes easily tolerate doses as high as $200 \mu \mathrm{M}$ TRG. ${ }^{16}$

Similar results were observed with K562 cells, as DOX selection dramatically increased MDR-1 protein levels (Figure 1B). Downregulation of MDR-1 in K562/DOX cells was specific to TRG, as rosiglitazone (ROSI) and pioglitazone (PIO) had no effect (Figure 1C). Interestingly, we could not detect BCRP expression in K562 cells regardless of whether the cells were selected or not (Figure 1D).

We recently discovered that TRG harbors histone deacetylase inhibitor activity (Davies and Harkness, unpublished data). To test whether TRG's downregulation of the $\mathrm{ABC}$ transporters could involve epigenetic changes, we asked whether another chromatin modifier, 5-azacytidine (5-AzaC), a DNA methylase inhibitor, could reduce MDR-1 and/or BCRP expression. Our results clearly demonstrate 
A

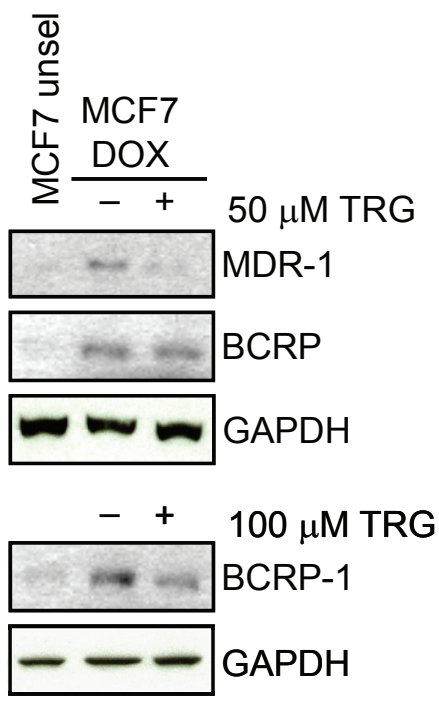

E

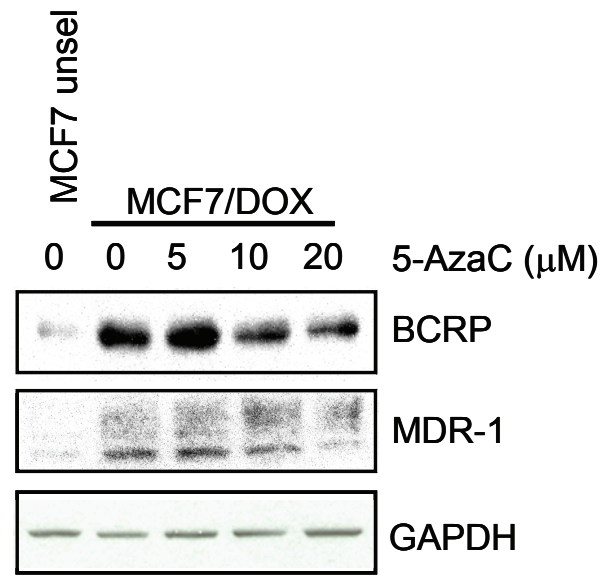

C

D
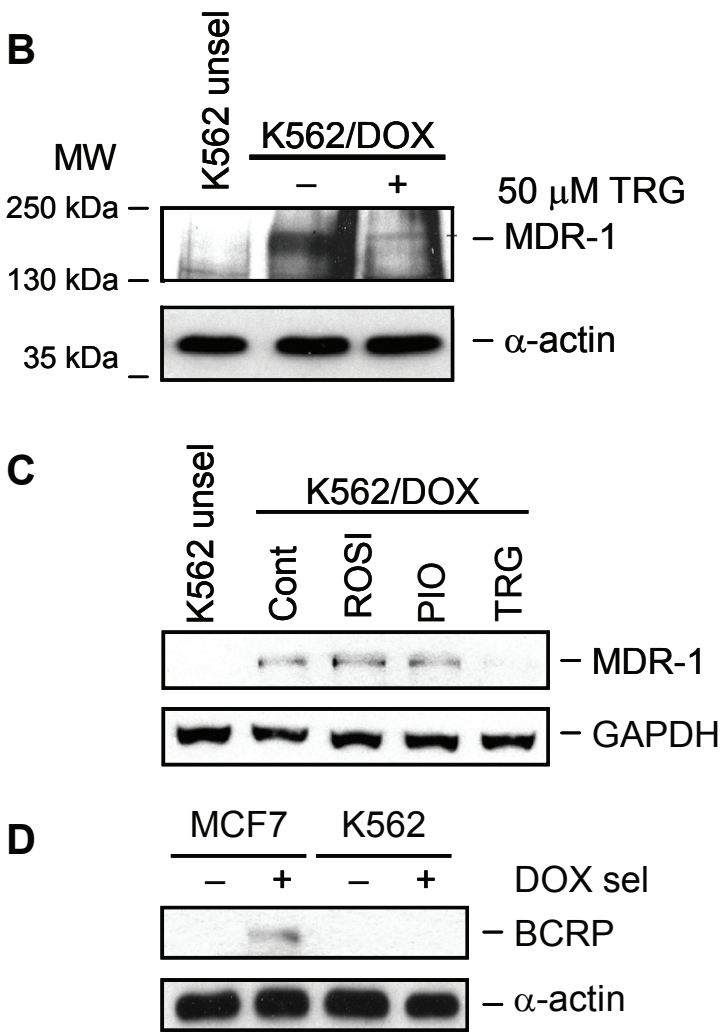

F

圈 Unselected

$\mathbf{\Delta}$ Selected

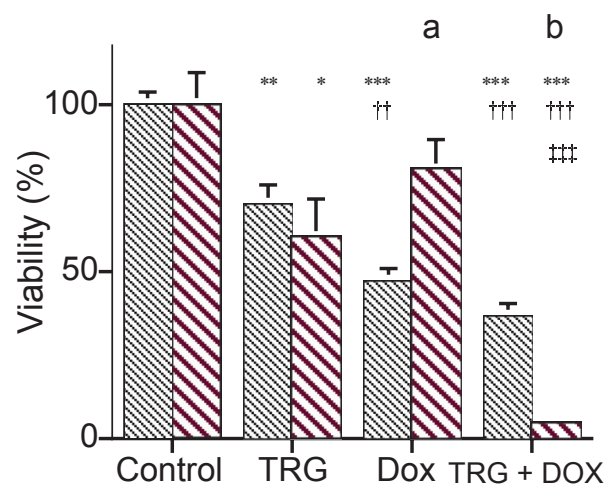

Figure I Troglitazone (TRG) and 5-azacytidine (5-AzaC) downregulate MDR-I and BCRP expression levels in doxorubicin (DOX)-resistant MCF7 breast cancer cells. A) RepresentativeWestern blots analyzing MDR-I and BCRP protein levels in lysates ( $100 \mu \mathrm{g} /$ lane for MDR-I and $60 \mu \mathrm{g} /$ lane for BCRP) of unselected and DOX-selected MCF7 cells. MCF7/DOX cells were treated with 50 or $100 \mu$ MTRG for $48 \mathrm{~h}$ prior to harvest. For determining the equivalency of the protein load the same Western blot membrane was stripped and reprobed with a GAPDH antibody. B) Parental K562 leukemia cells and K562 cells selected for DOX resistance were treated with $50 \mu M T R G$ for 48 h. Protein lysates were examined for MDR-I protein expression using Western blotting. Antibodies against $\alpha$-actin were used to determine equivalency of protein load. C) K562/DOX cells were treated with $50 \mu \mathrm{M}$ ROSI, PIO, or TRG for $48 \mathrm{~h}$ prior to cell harvest and protein lysate preparation. Unselected K562 cells and K562/DOX cells treated with the drug vehicle DMSO were used as controls. Lysates were analyzed with antibody against MDR-I or $\alpha$-actin as a protein load control. D) Protein lysates prepared from selected and unselected MCF7 and K562 cells were examined for BCRP expression using antibodies against BCRP. E) Parental MCF7 cells and MCF7/DOX cells were treated with the 5-AzaC concentrations shown for $48 \mathrm{~h}$. Protein lysates were examined for MDR-I and BCRP expression usingWestern blot analyses.Antibodies against GAPDH served as protein load controls. F) Graph depicting how TRG $(50 \mu \mathrm{M})$ and DOX $(1 \mu \mathrm{M})$ alone or in combination affect the viability of unselected $(n=5 / g r o u p)$ and DOX-selected $(n=10 / g r o u p)$ MCF7 cells. Statistical analyses were done on the different treatments within a group using an ANOVA with a post-hoc Tukey-Kramer multiple comparison tests. Asterisks indicate significant differences (*P $<0.05$; **P $<0.01$; $* * * \mathrm{P}<0.001$ ) from the respective control cells. Daggers indicate significant differences ( ${ }^{t+P}<0.01$; tttP $<0.001$ ) from the respective TRG-treated cells. Double daggers indicate significant differences ( $\$ \#$ P $<0.00 \mathrm{I}$ ) from the respective DOX-treated cells. Statistical analyses between the same treatment groups in the unselected and selected cells were done using a nonparametric two-tailed Mann-Whitney test.There is a significant difference in response of unselected cells to DOX compared to selected cells $(\mathrm{a} . \mathrm{P}=0.008)$ and in response of unselected cells to DOX plus TRG compared to selected cells $(\mathrm{b}$. $\mathrm{P}=0.0007)$.

Abbreviations: BCRP, breast cancer resistance protein; GAPDH, glyceraldehyde-3-phosphate dehydrogenase; MDR, multiple drug resistance; MDR-I, multiple drug resistance protein 1 . 
that 5-AzaC downregulated both MDR-1 and BCRP in a dose-dependent manner in MCF7/DOX cells (Figure 1C). Thus, downregulation of MDR-1 and BCRP in MCF7/DOX cells likely involves epigenetic changes, potentially within the promoters of these genes.

We had previously shown that TRG treatment of K562/ DOX cells resulted in restoration of sensitivity to DOX treatment. ${ }^{12}$ Hence, we asked whether downregulation of MDR-1 by TRG in MCF7/DOX cells correlated with restoration of DOX sensitivity. Before addressing this question we examined the response of unselected MCF7 cells to TRG, DOX and DOX plus TRG. All treatments (for a $48 \mathrm{~h}$ period) significantly increased cell death (Figure 1D). TRG at $50 \mu \mathrm{M}$ caused $30 \%$ of the cells to die $(\mathrm{P}<0.01)$, DOX at $1 \mu \mathrm{M}$ caused $53 \%$ of the cells to die $(\mathrm{P}<0.001)$ while TRG plus DOX caused 63\% of the cells to die ( $<<0.001)$. In DOX-selected cells the addition of TRG $(50 \mu \mathrm{M})$ for a $48 \mathrm{~h}$ period resulted in a significant $40 \%(\mathrm{P}<0.05)$ decrease in cell viability (Figure 1D). Although the addition of DOX $(1 \mu \mathrm{M})$ resulted in a decrease in cell viability, this was not significantly $(\mathrm{P}>0.05)$ different from the cultures treated with only the DMSO vehicle. Treating the DOX-selected cultures with both TRG $(50 \mu \mathrm{M})$ and DOX $(1 \mu \mathrm{M})$ resulted in a loss of $96 \%$ of the cells which is significantly different from the control cells ( $\mathrm{P}<0.001$ ), the cells treated only with TRG $(\mathrm{P}<0.001)$ and cells treated only with DOX $(\mathrm{P}<0.001)$.

To test whether TRG-dependent downregulation of MDR-1 is responsible for resensitizing MCF7/DOX cells to DOX, we treated MCF7/DOX cells with MDR-1 siRNA oligos. Our results show that MDR-1 siRNA does indeed reduce MDR-1 protein expression compared to cells treated with MDR-1 scrambled control oligos (Figure 2A). Next, MCF7/DOX cells treated with MDR-1 or scrambled siRNA oligos were treated with DOX for $48 \mathrm{~h}$, followed by MTT analysis to determine cell killing. The only significant killing of MCF7/DOX cells was observed with MDR-1 siRNA treament followed by DOX exposure (Figure 2B). These results suggest that reduction of MDR-1 alone is sufficient to render MCF7/DOX sensitive to DOX. TRG + DOX treatment had a greater effect on MCF/DOX cells than siRNA + $\mathrm{DOX}$, perhaps due to its broader range of $\mathrm{ABC}$ transporter downregulation as well as greater downregulation of MDR-1 (see Figure 1A).

TRG is a known PPAR $\gamma$ agonist. ${ }^{17,18}$ However, many recent reports demonstrate that TRG has PPAR $\gamma$-independent
A

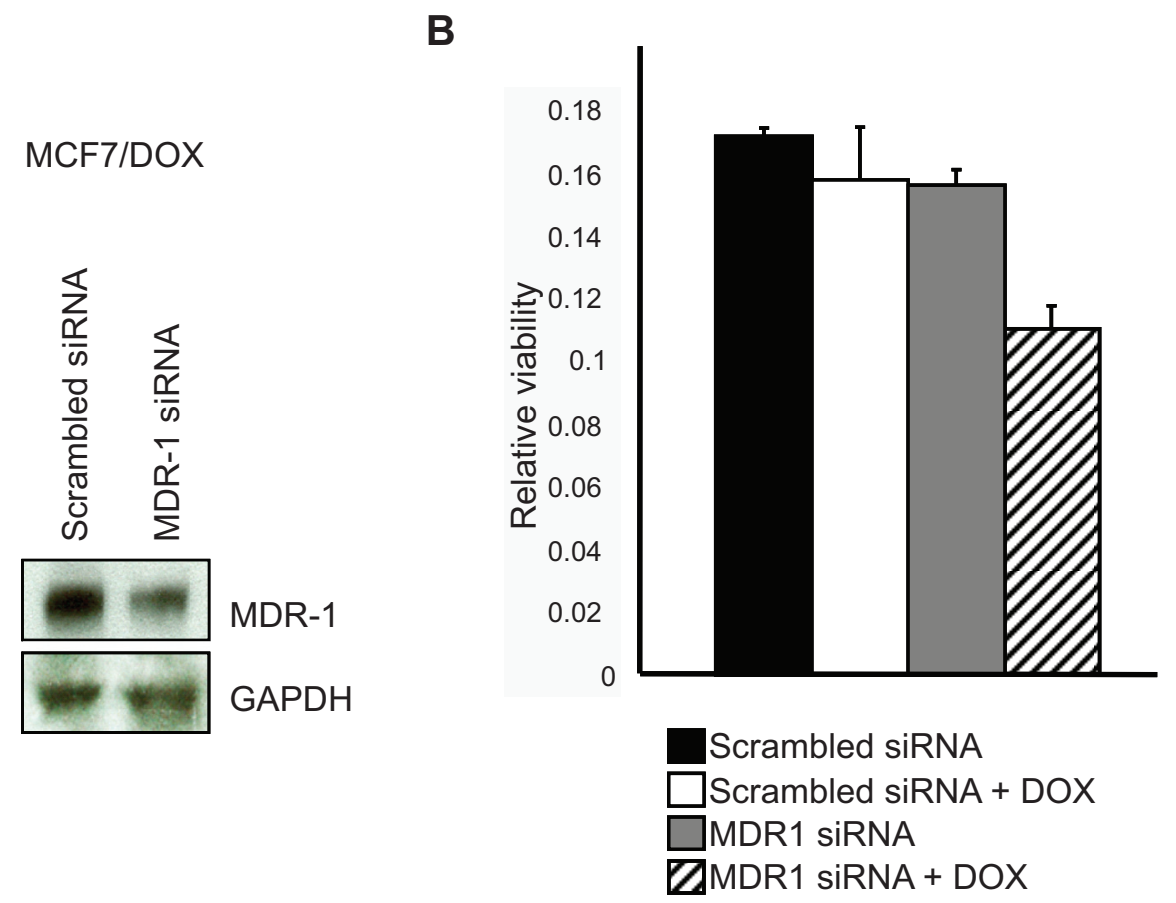

Figure 2 MDR-I gene silencing reduces MDR-I proteins levels and renders MCF7/DOX cells sensitive to DOX. A) MCF7/DOX cells were treated with MDR-I or scrambled siRNA oligos for 48 hrs. Protein lysates were prepared and analyzed using antibodies against MDR-I and GAPDH as a protein load control. B) Cell killing was determined by MTT analysis of MCF7/DOX cells treated as above. The analysis was repeated three times and standard error of the mean is shown. A statistical analysis was performed using ANOVA with a post-hoc Tukey-Kramer test. MDR-I siRNA + DOX is significantly different from control $(P<0.5)$.

Abbreviations: ANOVA, analysis of variance; DOX, doxorubicin; GAPDH, glyceraldehyde-3-phosphate dehydrogenase; MDR-I, multiple drug resistance protein I. 
activity by acting on uncharacterized off-targets. ${ }^{19,20}$ To examine whether TRG activity in MCF7 cells is PPAR $\gamma$ dependent, we exposed TRG-treated MCF7 parental cells to increasing doses of the PPAR $\gamma$ irreversible inhibitor GW9662. GW9662 inhibits PPAR $\gamma$ with an $\mathrm{IC}_{50}$ of $3.3 \mathrm{nM} .^{21}$ We tested the ability of GW9662 to block induction of histone H3 lysine 9 (H3K9) acetylation by TRG in parental MCF7 cells, which we demonstrated in $\mathrm{K} 562$ cells $^{12}$ and have since reproduced in multiple cell lines, including MCF7 cells (data not shown). We expected that if induction of $\mathrm{H} 3 \mathrm{~K} 9$ acetylation by TRG was PPAR $\gamma$-dependent, then inhibition of PPAR $\gamma$ using GW9662 should abolish this effect. Opposed to this prediction, GW9662 increased H3K9 acetylation in TRG-treated cells in a dose dependent manner beyond that observed with TRG (Figure 3A). This synergistic response suggests the novel hypothesis that PPAR $\gamma$ inhibition actually enhances TRG activities. Interestingly, GW9662 also induced
H3K79 methylation in MCF7 cells (Figure 3B), which we have observed with TRG treatment (Davies and Harkness, unpublished data). Methylation of H3K79 is associated with DNA damage, ${ }^{22,23}$ indicating that PPAR $\gamma$ may promote the health of MCF7/DOX cells and act counter to TRG.

To examine whether PPAR $\gamma$ antagonizes TRG action on BCRP expression in MCF7/DOX cells, we treated selected MCF7 cells with increasing doses of GW9662 and asked what effect this had on BCRP expression. Our results show that GW9662 alone could reduce BCRP expression (Figure 3C). Our results provide evidence that TRG and PPAR $\gamma$ may have an antagonistic interaction in MCF7 drug-resistant cells, perhaps by acting in opposite manner on MDR-1 expression.

Lastly, we tested whether down regulation of BCRP required distinct signaling pathways. We recently observed that induction of histone modifications by TRG and the
A

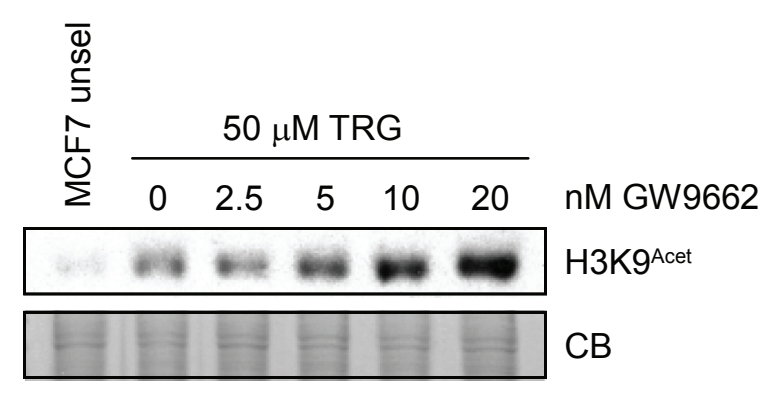

C

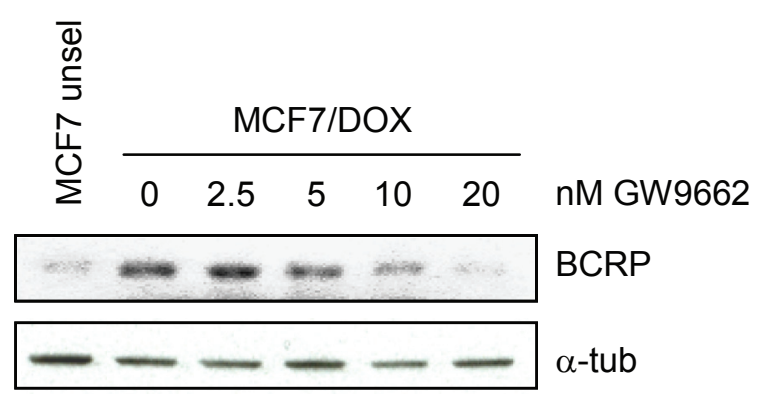

B
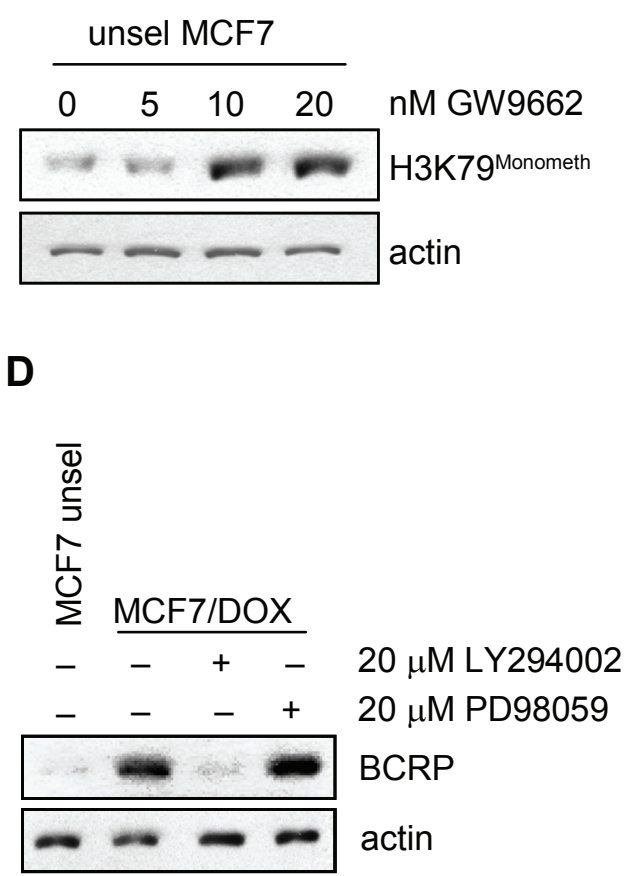

Figure 3 PPAR $\gamma$ inhibition accentuates TRG phenotypes in MCF7 cells. A) MCF7 parental cells were treated with $50 \mu M$ TRG or left untreated.To the TRG-treated cells, an increasing dose of GW9662 was added, as shown. The cells were incubated with the drugs for 48 h. Protein lysates were then prepared, separated by SDS PAGE and analyzed with antibody that specifically recognized acetylated histone H3 Lys9. A Coomassie Blue (CB)-stained gel is shown as a protein load control. B) Parental unselected MCF7 cells were treated with the same titration of GW9662 as shown in A.TRG was not used in this experiment. Protein lysates were examined with an antibody that specifically recognizes monomethylated histone $\mathrm{H} 3$ Lys79, a marker of DNA damage, that is also induced by TRG.Antibody against actin was used as protein load control. C) MCF7/DOX cells were treated with the GW9662 dose used above. Unselected control MCF7 cells were used as a control. Lysates were examined with antibodies against BCRP and $\alpha$-tubulin as a protein load control. D) Inhibition of AKT signaling downregulates BCRP expression. MCF7/DOX cells were treated with drugs that inhibit PI3K/AKT signaling, LY294002, or ERK signaling, PD98059, for 48 hours. Protein lysates were prepared and analyzed with antibody against BCRP.

Abbreviations: BCRP, breast cancer resistance protein; DOX, doxorubicin; SDS PAGE, sodium dodecyl sulfate polyacrylamide gel electrophoresis; TRG, troglitazone. 
histone acetyltransferases trichostatin A (TSA) and PXD101 depended on AKT inhibition (Davies and Harkness, unpublished data). Thus, we predicted that inhibition of AKT signaling, using the drug LY294002, would also downregulate BCRP expression. Our results confirm this prediction, as inhibition of AKT signaling reduced BCRP protein levels, but inhibition of ERK signaling, using PD98059, did not (Figure 3D).

Taken together, our data describes a mechanistic pathway leading to downregulation of the ABC transporters MDR-1 and BCRP in drug-resistant MCF7 cells. We suggest the novel antagonistic interplay between TRG and PPAR $\gamma$ in drug-resistant MCF7 cells. Our data supports the possibility that PPAR $\gamma$ plays a role in MDR-1 and/or BCRP expression and is required for the drug-resistant phenotype. Further work is planned to investigate this possibility. Our work also demonstrates that TRG's action on histone modifications and downregulation of ABC transporters may be tightly linked, as our experiments that alter histone modifications also affect MDR-1 and/or BCRP expression.

\section{Discussion}

In the present experiments we have shown that the development of DOX resistance in both MCF7 and K562 cells following DOX-selection is associated with increased levels of the ABC transporters MDR-1 and BCRP. Expression of BCRP was previously demonstrated in a DOX-resistant MCF7 cell line. ${ }^{24}$ Exposure of MCF7/DOX and K562/DOX cells to TRG resulted in decreased levels of both MDR-1 and BCRP. Furthermore, we show that following exposure to TRG, the DOX-selected cells again become susceptible to DOX treatment. This is similar to our previous observations showing DOX-selected K562 human leukemia cells exposed to both TRG and DOX were killed more readily. ${ }^{12}$ In that study, TRG also downregulated GLO-1 expression in $\mathrm{K} 562 / \mathrm{DOX}$ cells. Increased GLO-1 expression is thought to be associated with the MDR phenotype..$^{10,11}$ Our combined findings support the hypothesis that TRG induces multiple changes within MDR cells that lead to increased sensitivity to DOX once again.

TRG is more effective at downregulating MDR-1 than BCRP (Figure 1A). BCRP does not efflux anthracyclines such as DOX unless there is a mutation within codon 482 of the BCRP gene. ${ }^{24}$ Thus, downregulation of MDR-1 gene expression likely accounts, in part, for how TRG overcomes DOX-resistance. Our data supports this hypothesis, as partial silencing of MDR-1 using siRNA renders MCF7/DOX cells sensitive to DOX (Figure 2). Of interest is that the combination of TRG and DOX was much more effective at killing MCF7/DOX cells than in killing unselected MCF7 cells (Figure 1D). This may be due to a number of possible reasons. One we favor may be that in MCF7/DOX cells treated with TRG and DOX, DOX now accumulates because of the TRG-induced decreased expression of the ABC transporters. MDR cells display a wide range of alterations, including increased mutation to $\mathrm{p} 53$, reduced cell cycle checkpoint response and reduced apoptotic clearing of damaged cells. ${ }^{3}$ We have also found that TRG induces DNA damage (Davies and Harkness, unpublished data). The combination of increased DOX activity within these cells, and altered cell cycle control and cellular maintenance systems often observed in MDR cancer cells, suggests that MCF7/DOX cells cannot respond as effectively to DOX as unselected MCF7 cells. A second way that combination $\mathrm{DOX}+\mathrm{TRG}$ acts to reverse DOX resistance may be due to selection of epigenetic changes that make them more vulnerable to antiproliferative agents. We have previously shown that DOX-selected K562 cells have major changes in histone $\mathrm{H} 3$ acetylation and phosphorylation. ${ }^{12}$ DOX-resistant MCF7 cells have also been shown to have major epigenetic changes including global decreases in DNA cytosine methylation, gene-specific hypo- or hypermethylation states as well as changes in histone acetylation and methylation states. ${ }^{25}$ Our unpublished work shows that TRG can inhibit the activity of histone acetyltransferases (HDACs) in treated cells, and more importantly, in treated cell lysates, which suggests that TRG is capable of binding HDAC enzymes to block their function.

There are at least two possible approaches in countering MDR. One is the development of compounds that interfere with the drug efflux mechanism of the $\mathrm{ABC}$ transporters $^{26}$ and clinical trials on the most promising candidates have, so far, led to disappointing outcomes. ${ }^{27}$ A major problem with using transport inhibitors is their nonspecificity for cancer cells. These efflux pumps have been found, and have functions, in a variety of tissues. Thus, for example, MDR-1 is strongly expressed in the adrenal gland, kidney and small intestine whereas BCRP is strongly expressed in tissues such as in liver and central nervous system: ${ }^{28}$ hence, inhibitors can affect normal physiological function. A second approach is to downregulate the expression of the drug efflux pumps. One way to do this is by using molecular biology approaches, for example, delivery of siRNAs. ${ }^{29}$ How readily this can be translated into human clinical trials is not clear, as siRNA delivery must be selective for tumor cells, otherwise, normal physiological functions may be affected. In addition, the 
delivery vector should not give rise to ontoward effects. The use of a pharmacological approach to downregulate MDR-1 expression just prior to chemotherapy seems a more feasible approach. A number of compounds have been shown to partially decrease MDR-1 expression, these include honokiol (2-(4-hydroxy-3-prop-2-enyl-phenyl)4-prop-2-enyl-phenol), a biphenolic compound present in traditional Japanese medicines prepared from Magnolia grandifloris $^{30}$ and curcumin $((1 E, 6 E)$-1,7-bis(4-hydroxy3-methoxyphenyl)-1,6-heptadiene-3,5-dione), a biphenolic that is the major anticancer compound found in turmeric. ${ }^{31}$ Recently the histone deacetylase inhibitors (HDACi's) Trichostatin A (TSA) and sodium butyrate have been shown to downregulate MDR-1 expression in multidrug-resistant small cell lung carcinoma cell line. ${ }^{32}$ These data support our hypothesis that MDR-1 and BCRP downregulation by TRG involves inhibition of HDAC enzymes and epigenetic alterations at these promoters.

We took several approaches to examine possible mechanisms involved in MDR-1 and BCRP downregulation. First, we tested whether the DNA methylase inhibitor 5-AzaC could also downregulate MDR-1 and/or BCRP in MCF7/DOX cells. We found that 5-AzaC could indeed downregulate both BCRP and MDR-1 in a dose-dependent manner (Figure 1C). This is in contrast to observations that 5-AzaC treatment of human drug sensitive $\mathrm{T}$ cell leukemia cells resulted in hypomethylation of the MDR-1 promoter and activation of transcription. ${ }^{33}$ Similarly, in cultured human keratinocytes, $7.5 \mu \mathrm{M}$ TRG induced transcription of the $\mathrm{ABC}$ transporter family member ABCA12, mutation of which is responsible for type 2 lamellar ichthyosis and Harlequin ichthyosis. ${ }^{34}$ The promoter of MDR-1 in DOX-resistant MCF7 was previously shown to harbor hypomethylated DNA and hyperacetylated histones, consistent with transcriptional activation. ${ }^{35}$ Thus, downregulation of MDR-1 in MCF7/DOX by 5-AzaC in our experiments likely occurs via increased activity of a transcriptional repressor that acts at the MDR-1 promoter. Interestingly, a previous study of drugresistant K562 cells demonstrated that the repressor binding site within the MDR-1 promoter was methylated, blocking binding of a repressor. ${ }^{36}$ Treatment of these cells with 5-AzaC demethylated the repressor binding site, leading to downregulated transcription of MDR-1, a beneficial outcome. Several proteins have been shown to repress MDR-1 expression, including the AML1/ETO and TEL/AML1 chimeric proteins found in acute lymphoblastic leukemia (ALL), ${ }^{37}$ the pathogenic amino-terminal region of huntingtin (htt), httex $1 \mathrm{p},{ }^{38}$ and c-Jun. ${ }^{39}$ The repression of MDR-1 expression by c-Jun is stimulated by a ubiquitin-protein ligase, Siah1, that conversely does not promote MDR-1 degradation, but rather, increases its transcriptional downregulation by promoting the binding of the c-Jun repressor to the AP1 activator binding site in the MDR-1 promoter. ${ }^{40}$ The action of c-Jun likely plays a key role in mediating 5-AzaC, and perhaps TRG, down-regutation of at least MDR-1.

A second possible mechanism accounting for downregulation of the ABC transporters could involve PPAR $\gamma$. TRG is a known PPAR $\gamma$ ligand and has been shown in previous studies to act as a PPAR $\gamma$ agonist. ${ }^{17,18}$ Recently, many studies have indicated TRG may have "off-targets" that are not PPAR $\gamma$ dependent. ${ }^{19,20}$ Our studies show that use of the irreversible PPAR $\gamma$ inhibitor GW9662 in the nM range (5-20 nM) does not block TRG effects, but rather, accentuates them. For example, GW9662 increased histone H3K9 acetylation in TRG-treated parental MCF7 cells (Figure 3A); increased histone $\mathrm{H} 3 \mathrm{~K} 79$ monomethylation in untreated MCF7 parental cells (Figure 3B); and reduced expression of BCRP in MCF7/ DOX cells (Figure 3C). Increased histone modifications are observed in MCF7 (Davies and Harkness, unpublished data) and K562 cells ${ }^{12}$ when treated with TRG. Thus, PPAR $\gamma$ may be acting in an opposing fashion to TRG. A recent report found that GW9662 was a potent antiproliferative agent against hematopoietic and epithelial cancer cell lines and that these effects did not correlate with PPAR $\gamma$ protein levels. ${ }^{41}$ It was concluded that GW9662 may have other mechanisms of action that may be PPAR $\gamma$-independent. However, it is important to note that GW9662 in these studies was used in the $\mu \mathrm{M}$ range $(5-25 \mu \mathrm{M}), 1,000 \times$ more concentrated than the concentrations used in our studies. Leesnitzer and colleagues $^{21}$ showed that GW9662 inhibited PPAR $\gamma, \operatorname{PPAR} \alpha$, and PPAR $\delta$ and with $\mathrm{IC}_{50} \mathrm{~s}$ of $3.3 \mathrm{nM}, 32 \mathrm{nM}$, and $2 \mu \mathrm{M}$, respectively. Furthermore, Leesnitzer and colleagues ${ }^{21}$ show that at $10 \mu \mathrm{M}$, GW9662 acted as an agonist for the nuclear receptors PXR and FXR. In the $\mu \mathrm{M}$ range GW9662 kills cells and shows action on multiple "off-targets". ${ }^{21,41,42}$ In our studies presented here using GW9662 in the nM range, we do not observe killing, but do observe multiple effects at the histone and BCRP protein level. Nonetheless, recent literature implicates PPAR $\gamma$ as an antiproliferative agent, not one involved in promoting an MDR phenotype. ${ }^{43-45}$ Thus, additional work must be conducted to determine whether the effects of GW9662 in our study implicate PPAR $\gamma$ as a mediator of MDR.

Recently we observed that TRG killing of cancer cells is associated with decreased AKT phosphorylation (Davies and Harkness, unpublished data). Treatment of MCF7 
cells with the PI3 kinase inhibitor LY294002, but not the ERK inhibitor PD98059, resulted in increased H3K9 acetylation, similar to TRG and trichostatin A treatment. Here, we show that LY294002, and not PD98059, downregulated BCRP expression (Figure 3D). The status of TRG and its action on AKT signaling is currently controversial, as recent reports show that TRG either plays or does not play a role in AKT function. ${ }^{46-50}$ Our data support the idea that AKT signaling plays a role in the MDR phenotype.

Of the experimental approaches that have shown to downregulate MDR, TRG has the major advantage in that there is extensive clinical experience using TRG as an insulinsensitizing agent for treatment of type 2 diabetes. ${ }^{51}$ TRG was withdrawn from the market because chronic treatment resulted in a small subset of patients $(<2 \%)$ experiencing severe liver dysfunction that occasionally led to death due to liver failure (1:100,000 patients monitored; Scheen, 2001). ${ }^{52}$ However, if TRG were to be used as an adjunct therapy it would be given for only a few days before a chemotherapy session. Under such acute use one would not anticipate a similar occurrence of liver dysfunction observed with chronic TRG use.

Based upon an extensive literature demonstrating that TRG inhibits tumor growth in vitro and in animal models, ${ }^{53,54}$ human clinical trials were initiated where TRG was used as the sole chemotherapeutic agent. Unfortunately, these clinical trials have yielded disappointing results. ${ }^{55,56}$ Our data suggest that by downregulating expression of MDR-1 and BCRP in DOX-resistant cells, TRG ought to be a useful adjunct therapy in combination with a standard chemotherapy.

\section{Acknowledgments}

This research was supported by the Canadian Breast Cancer Foundation (Prairies, NWT) grant to Troy AA Harkness and Bernhard HJ Juurlink. We thank Dr T Arnason for a careful reading of the manuscript.

\section{References}

1. Levine MN, Whelan T. Adjuvant chemotherapy for breast cancer-30 years later. N Engl J Med. 2006;355:1920-1922.

2. Mayer EL, Burstein HJ. Chemotherapy for metastatic breast cancer. Hematol Oncol Clin North Am. 2007;21:257-272.

3. Coley HM. Mechanisms and strategies to overcome chemotherapy resistance in metastatic breast cancer. Cancer Treat Rev. 2008;34:378-390.

4. O'Driscoll L, Clynes M. Biomarkers and multiple drug resistance in breast cancer. Curr Cancer Drug Targets. 2006;6:365-384.

5. Mechetner E, Kyshtoobayeva A, Zonis S, et al. Levels of multidrug resistance (MDR1) P-glycoprotein expression by human breast cancer correlate with in vitro resistance to taxol and doxorubicin. Clin Cancer Res. 1998;4:389-398.

6. Atalay C, Deliloglu Gurhan I, Irkkan C, et al. Multidrug resistance in locally advanced breast cancer. Tumor Biol. 2006;27:309-318.
7. Borowski E, Bontemps-Gracz MM, Piwkowska A. Strategies for overcoming ABC-transporters-mediated multidrug resistance (MDR) of tumor cells. Acta Biochim Pol. 2005;52:609-627.

8. Perez-Tomas R. Multidrug resistance: retrospect and prospects in anticancer drug treatment. Curr Med Chem. 2006;13:1859-1876.

9. Wu L, Eftekharpour E, Davies GF, et al. Troglitazone selectively inhibits glyoxalase I gene expression. Diabetologia. 2001;44:2004-2012.

10. Sakamoto H, Mashima T, Kizaki A, et al. Glyoxalase I is involved in resistance of human leukemia cells to antitumor agent-induced apoptosis. Blood. 2000;95:3214-3218.

11. Rulli A, Carli L, Romani R, et al. Expression of glyoxalase I and II in normal and breast cancer tissues. Breast Cancer Res Treat. 2001;66:67-72.

12. Davies GF, Roesler WJ, Juurlink BH, Harkness TA. Troglitazone overcomes doxorubicin-resistance in resistant K562 leukemia cells. Leuk Lymphoma. 2005;46:1199-1206.

13. Bradford MM. A rapid and sensitive method for the quantitation of microgram quantities of protein using the principle of protein-dye binding. Analyt Biochem. 1976;72:248-254.

14. McHale AP, McHale L. Use of a tetrazolium based colorimetric assay in assessing photoradiation therapy in vitro. Cancer Lett. 1988;41:315-321.

15. Young PW, Buckle DR, Cantello BC, et al. Identification of high-affinity binding sites for the insulin sensitizer rosiglitazone (BRL-49653) in rodent and human adipocytes using a radioiodinated ligand for peroxisomal proliferator-activated receptor gamma. J Pharmacol Exp Ther. 1998;284:751-759.

16. Davies GF, Khandelwal RL, Wu L, et al. Inhibition of phosphoenolpyruvate carboxykinase (PEPCK) gene expression by troglitazone: a peroxisome proliferator-activated receptor-gamma (PPARgamma)independent, antioxidant-related mechanism. Biochem Pharmacol. 2001;62:1071-1079.

17. Giannini S, Serio M, Galli A. Pleiotropic effects of thiazolidinediones: taking a look beyond antidiabetic activity. J Endocrinol Invest. 2004;27:982-991.

18. Ríos-Vázquez R, Marzoa-Rivas R, Gil-Ortega I, Kaski JC. Peroxisome proliferator-activated receptor-gamma agonists for management and prevention of vascular disease in patients with and without diabetes mellitus. Am J Cardiovasc Drugs. 2006;6:231-242.

19. Seufert S, Coras R, Tränkle C, et al. PPAR gamma activators: Offtarget against glioma cell migration and brain invasion. PPAR Res. 2008;513943.

20. Yamashita M. PPARalpha/gamma-independent effects of PPARalpha/ gamma ligands on cysteinyl leukotriene production in mast cells. PPAR Res. 2008;29:35-38.

21. Leesnitzer LM, Parks DJ, Bledsoe RK, et al. Functional consequences of cysteine modification in the ligand binding sites of peroxisome proliferator activated receptors by GW9662. Biochemistry. 2002;41:6640-6650.

22. Zhou H, Madden BJ, Muddiman DC, Zhang Z. Chromatin assembly factor 1 interacts with histone $\mathrm{H} 3$ methylated at lysine 79 in the processes of epigenetic silencing and DNA repair. Biochemistry. 2006;45:2852-2861.

23. Bostelman LJ, Keller AM, Albrecht AM, et al. Methylation of histone $\mathrm{H} 3$ lysine-79 by Dot1p plays multiple roles in the response to UV damage in Saccharomyces cerevisiae. DNA Repair. 2007;6:383-395.

24. Doyle LA, Ross DD. Multidrug resistance mediated by the breast cancer resistance protein BCRP (ABCG2). Oncogene. 2003;22:7340-7358.

25. Chekhun VF, Lukyanova NY, Kovalchuk O, et al. Epigenetic profiling of multidrug-resistant human MCF-7 breast adenocarcinoma cells reveals novel hyper- and hypomethylated targets. Mol Cancer Ther. 2007;6:1089-1098.

26. McDevitt CA, Callaghan R. How can we best use structural information on P-glycoprotein to design inhibitors? Pharmacol Ther. 2007;113:429-441.

27. Nobili S, Landini I, Giglioni B, et al. Pharmacological strategies for overcoming multidrug resistance. Curr Drug Targets. 2006;7:861-879. 
28. Nishimura M, Naito S. Tissue-specific mRNA expression profiles of human ATP-binding cassette and solute carrier transporter superfamilies. Drug Metab Pharmacokinet. 2005;20:452-477.

29. Kaszubiak A, Holm PS, Lage H. Overcoming the classical multidrug resistance phenotype by adenoviral delivery of anti-MDR1 short hairpin RNAs and ribozymes. Int J Oncol. 2007;31:419-430.

30. Xu D, Lu Q, Hu X. Down-regulation of P-glycoprotein expression in MDR breast cancer cell MCF-7/ADR by honokiol. Cancer Lett. 2006;243:274-280.

31. Anuchapreeda S, Leechanachai P, Smith MM, et al. Modulation of P-glycoprotein expression and function by curcumin in multidrug-resistant human KB cells. Biochem Pharmacol. 2002;64:573-582.

32. El-Khoury V, Breuzard G, Fourre N, et al. The histone deacetylase inhibitor trichostatin A down-regulateshuman MDR1 (ABCB1) gene expression by a transcription-dependent mechanism in a drugresistant small cell lung carcinoma cell line model. Br J Cancer. 2007;97:562-573.

33. Kantharidis P, El-Osta A, deSilva M, et al. Altered methylation of the human MDR1 promoter is associated with acquired multidrug resistance. Clin Cancer Res. 1997;3:2025-2032.

34. Jiang YJ, Lu B, Kim P, et al. PPAR and LXR activators regulate ABCA12 expression in human keratinocytes. J Invest Dermatol. 2008;128:104-109.

35. David GL, Yegnasubramanian S, Kumar A, et al. MDR1 promoter hypermethylation in MCF-7 human breast cancer cells: changes in chromatin structure induced by treatment with 5-Aza-cytidine. Cancer Biol Ther. 2004;3:540-548.

36. Ando T, Nishimura M, Oka Y. Decitabine (5-Aza-2'-deoxycytidine) decreased DNA methylation and expression of MDR-1 gene in K562/ ADM cells. Leukemia. 2000;14:1915-1920.

37. Asakura K, Uchida H, Miyachi H, et al. TEL/AML1 overcomes drug resistance through transcriptional repression of multidrug resistance-1 gene expression. Mol Cancer Res. 2004;2:339-347.

38. Steffan JS, Kazantsev A, Spasic-Boskovic O, et al. The Huntington's disease protein interacts with $\mathrm{p} 53$ and CREB-binding protein and represses transcription. Proc Natl Acad Sci U S A. 2000;97:6763-6768.

39. Miao ZH, Ding J. Transcription factor c-Jun activation represses mdr-1 gene expression. Cancer Res. 2003;63:4527-4532.

40. Liu M, Aneja R, Wang H, et al. Modulation of multidrug resistance in cancer cells by the E3 ubiquitin ligase seven-in-absentia homologue 1. J Pathol. 2008;214:508-514.

41. Burton JD, Castillo ME, Goldenberg DM, Blumenthal RD. Peroxisome proliferator-activated receptor-gamma antagonists exhibit potent antiproliferative effects versus many hematopoietic and epithelial cancer cell lines. Anticancer Drugs. 2007;18:525-534.

42. Cheon $\mathrm{CW}, \mathrm{Kim} \mathrm{DH}, \mathrm{Kim} \mathrm{DH}$, et al. Effects of ciglitazone and troglitazone on the proliferation of human stomach cancer cells. World J Gastroenterol. 2009;15:310-320.
43. Wang LH, Yang XY, Zhang X, Farrar WL. Inhibition of adhesive interaction between multiple myeloma and bone marrow stromal cells by PPARgamma cross talk with NF-kappaB and C/EBP. Blood. 2007;110:4373-4384.

44. Brodbeck J, Balestra ME, Saunders AM, et al. Rosiglitazone increases dendritic spine density and rescues spine loss caused by apolipoprotein E4 in primary cortical neurons. Proc Natl Acad Sci USA. 2008;105:1343-1346.

45. Zand H, Rahimipour A, Salimi S, Shafiee SM. Docosahexaenoic acid sensitizes Ramos cells to Gamma-irradiation-induced apoptosis through involvement of PPAR-gamma activation and NF-kappaB suppression. Mol Cell Biochem. 2008;317:113-120.

46. Planavila A, Alegret M, Sánchez RM, et al. Increased Akt protein expression is associated with decreased ceramide content in skeletal muscle of troglitazone-treated mice. Biochem Pharmacol. 2005;69:1195-1204.

47. Kim YK, Choi HY, Kim NH, et al. Reversine stimulates adipocyte differentiation and downregulates Akt and p70(s6k) signaling pathways in 3T3-L1 cells. Biochem Biophys Res Commun. 2007;358:553-558.

48. Yang YC, Ho TC, Chen SL, et al. Inhibition of cell motility by troglitazone in human ovarian carcinoma cell line. BMC Cancer. 2007; 7:216

49. Fediuc S, Pimenta AS, Gaidhu MP, Ceddia RB. Activation of AMP-activated protein kinase, inhibition of pyruvate dehydrogenase activity, and redistribution of substrate partitioning mediate the acute insulin-sensitizing effects of troglitazone in skeletal muscle cells. J Cell Physiol. 2008;215:392-400.

50. Wang PS, Chou FS, Bloomston M, et al. Thiazolidinediones downregulate wnt/beta-catenin signaling via multiple mechanisms in breast cancer cells. J Surg Res. 2008;Jun 27 [Epub ahead of print].

51. Saltiel AR, Olefsky JM. Thiazolidinediones in the treatment of insulin resistance and type II diabetes. Diabetes. 1996;45:1661-1669.

52. Scheen AJ. Thiazolidinediones and liver toxicity. Diabetes Metab. 2001;27:305-313.

53. Kawa S, Nikaido T, Unno H, et al. Growth inhibition and differentiation of pancreatic cancer cell lines by PPAR gamma ligand troglitazone. Pancreas. 2002;24:1-7.

54. Yu J, Qiao L, Zimmermann L, et al. Troglitazone inhibits tumor growth in hepatocellular carcinoma in vitro and in vivo. Hepatology. 2006;43:134-143.

55. Kulke MH, Demetri GD, Sharpless NE, et al. A phase II study of troglitazone, an activator of the PPARgamma receptor, in patients with chemotherapy-resistant metastatic colorectal cancer. Cancer J. 2002;8:395-399.

56. Burstein HJ, Demetri GD, Mueller E, et al. Use of the peroxisome proliferator-activated receptor (PPAR) gamma ligand troglitazone as treatment for refractory breast cancer: a phase II study. Breast Cancer Res Treat. 2003;79:391-397. 